\title{
Simulations of imaging extended sources using the GMRT and the U-GMRT
}

\section{Implications to observing strategies}

\author{
Deepak Kumar Deo · Ruta Kale
}

Received: date / Accepted: date

\begin{abstract}
Astrophysical sources such as radio halos and relics in galaxy clusters, supernova remnants and radio galaxies have angular sizes from a few to several 10 s of arcminutes. In radio interferometric imaging of

rations. The presented simulations can be carried out for future radio telescopes such as the Square Kilometre Array (SKA) for optimisation of observing strategies to image extended radio sources.
\end{abstract} such sources, the largest angular size of the source that can be imaged is limited by the shortest projected baseline towards the source. It is essential to determine the limitations of the recovery of the extended features on various angular scales in order to interpret the radio image. We simulated observations of a model extended source of Gaussian shape with the Giant Metrewave Radio Telescope (GMRT) using Common Astronomy Software Applications (CASA). The recovery in flux density and in morphology of the model source was quantified in a variety of observing cases with changing source properties and the uv-coverage. If $\theta_{\text {lar }}$ is the largest angular scale sampled in an observation with the GMRT, then $>80 \%$ recovery of a source of size $0.3 \times \theta_{\text {lar }}$ is possible. The upgraded GMRT (U-GMRT) providing $200 \mathrm{MHz}$ instantaneous bandwidth between $300-500 \mathrm{MHz}$ will allow a factor of two better recovery of a source of size $\theta_{\text {lar }}$ as compared to the GMRT at $300 \mathrm{MHz}$ with $33 \mathrm{MHz}$ bandwidth. We provide quantitative estimates for the improvement in extended source recovery in observations at low elevations and long du-

Deepak Kumar Deo

National Centre for Radio Astrophysics, Tata Institute of Fundamental Research, Post Bag 3, Pune University Campus, Pune 411007, India

Tel.: +91-9039748525

E-mail: anudeo.5@gmail.com

Ruta Kale

National Centre for Radio Astrophysics, Tata Institute of Fundamental Research, Post Bag 3, Pune University Campus, Pune 411007, India

Tel.: +91-20-25719234

E-mail: ruta@ncra.tifr.res.in

Keywords Radio interferometers - GMRT - imaging - observation simulation - CASA

\section{Introduction}

Imaging of astrophysical sources with extended features like supernova remnants, star-forming regions, radio galaxies and radio halos and relics in galaxy clusters is important as we can infer morphology, spectrum and polarisation of the source. These can reveal the underlying physical mechanisms at work in these sources which in turn, would help us to understand the evolution of our universe in greater detail.

Radio interferometers measure the Fourier transform of the sky at the spatial frequencies defined by the configuration of the telescope elements (antennas). The quality of an image from a radio interferometer depends on the distribution of uv-coverage which is the set of all baseline vectors projected on a plane perpendicular to the source direction (called as uv-plane) during the observations. Fourier transform of the uv-coverage is called as the 'dirty beam'. The radio interferometer data contains the sky distribution convolved with the dirty beam and deconvolution is used to get the actual sky distribution. The more filled and uniform is the uvcoverage, the better would be the shape of the dirty beam and hence a better image.

For a radio interferometer with $\mathrm{N}$ antennas, there are ${ }^{N} C_{2}$ number of baselines. The uv-coverage will span between the shortest and the longest baselines $\left(B_{\min }\right.$ and $\left.B_{\max }\right)$ in a given observation towards a target and 
by earth rotation synthesis, each baseline vector will trace elliptical tracks in the uv-plane [13]. The angular scales sampled in the sky distribution correspond to the spatial frequencies sampled in the uv-plane. The shortest length in the uv-plane will limit the largest angular scale sampled in the sky $\left(\sim \lambda / B_{\text {min }}\right)$ and the longest baseline will limit the angular resolution $\left(\sim \lambda / B_{\max }\right)$ of the radio interferometer. Sources in the sky can have structures ranging from scales smaller than the resolution of the interferometers and larger than the largest sampled angular scale. Since the sky distribution is unknown, it is essential to determine the limitations of the recovery of the sky distribution on various angular scales in order to interpret the radio image.

The problem is severe in the cases of extended sources such as molecular clouds, nebulae and sources called the radio halos associated with galaxy clusters where the angular extents of the sources (e.g. several tens of arcminutes) are often larger than the largest angular scale sampled by the radio interferometer. A one-dimensional (1D) simulation study was done by Wilner and Welch [17] on quantifying the effect of holes in the uv-plane on the imaging of molecular clouds. They modelled a 1D Gaussian and uniform disk source distribution as a representation of extended molecular emissions and derived the expressions to quantify the effect of holes in their imaging. They found that the central brightness recovered from a Gaussian distribution characterised by FWHM $\sim \lambda / B_{\min }$ is only about $3 \%$. They also found that the central hole in the uv-coverage gives rise to more significant image distortions than many scattered outer holes.

The Giant Metrewave Radio Telescope (GMRT), the Very Large Array (VLA), Westerbork Synthesis Radio Telescope (WSRT) and the Australia Telescope Compact Array (ATCA) are among the largest radio interferometers that are used extensively to observe the sky in radio frequency bands. These are radio interferometers with dish type elements. The VLA, WSRT and ATCA have one or more movable elements to increase the uv-coverage. The GMRT is a telescope with a fixed configuration with elements distributed in a hybrid configuration providing good uv-coverage at short and long baselines. It is among the few telescopes operating at frequencies $<1 \mathrm{GHz}$ and has been extensively used to observe extended radio sources such as radio halos in galaxy clusters [e.g 7, 16]. In case of surveys that target search of extended sources such as radio halos, putting upper limits on non-detections requires a procedure of injection of model radio halos and testing their recovery in the given dataset. This method has been successfully used in the GMRT Radio Halo Survey [GRHS 14, 15] and its extension, the EGRHS
$[9,8]$. The underlying issue is that the imaging of extended emission is highly sensitive to the uv-coverage in a given observation. Non-availability of some antennas in the array, corruption due to radio frequency interference can modify the uv-coverage significantly and lead poorer sensitivity to extended emission.

The next generation instruments such as the Karl G. Jansky VLA (JVLA) and the upgraded GMRT (UGMRT) are moving towards wide bandwidth receivers which will drastically improve the uv-coverage. A comparison of the new facilities with the old ones is necessary to be quantified to understand the strengths of the new systems.

In this study, we quantify the effect of uv-coverage on the recovery of the total flux density and morphology of an extended radio source using simulated GMRT observations of model extended sources. We studied the recovery as a function of the angular size of the source, position in the sky, observation duration for the GMRT. We also simulate observations with the U-GMRT and compare it with the GMRT.

The paper is organised as follows: Sec. 2 gives an overview of the simulation, Sec. 3 presents the results which are discussed in Sec. 4. Summary and conclusions are presented in Sec. 5 .

\subsection{GMRT and U-GMRT}

Giant Metrewave Radio Telescope (GMRT) [12] is a radio interferometer set up by NCRA-TIFR at a site about $80 \mathrm{~km}$ north of Pune, India. GMRT (Latitude $=19.1^{\circ} \mathrm{N}$, Longitude $=74.05^{\circ} \mathrm{E}$ ) consists of thirty $45 \mathrm{~m}$ diameter antennas spread over a region of $25 \mathrm{~km}$ diameter. The antennas are distributed in a hybrid configuration, 14 of which are located in a central compact array of size $\sim 1.1 \mathrm{~km}$ and the rest 16 are distributed in a roughly ' $\mathrm{Y}$ ' shaped configuration (Fig. 1), giving a maximum baseline length of $\sim 25 \mathrm{~km}$. GMRT can observe in a declination range of $-53^{\circ}$ to $90^{\circ}$. It provides a maximum instantaneous bandwidth of $32 \mathrm{MHz}$ for observation and can be used for observations between $130 \mathrm{MHz}$ to 1450 $\mathrm{MHz}$ in the bands $130-170 \mathrm{MHz}, 225-245 \mathrm{MHz}, 300-360$ $\mathrm{MHz}, 580-660 \mathrm{MHz}$ and $1000-1450 \mathrm{MHz}[1,2]$. We have used the $610 \mathrm{MHz}$ GMRT band as a fiducial band for simulations to quantify the limitations of recovering an extended radio source.

The upgraded GMRT (U-GMRT) will have new wide band receivers that will allow instantaneous bandwidth of $200 \mathrm{MHz}$ in the frequency bands 120 - 250, 250 - 500 and $550-850 \mathrm{MHz}$ and of $400 \mathrm{MHz}$ in the $1050-1450$ $\mathrm{MHz}$ band $[3,2]$. The broadband receivers will allow a drastic increase in the uv-coverage. In this work we 
compare the current GMRT and the U-GMRT in the $250-500 \mathrm{MHz}$ band in terms of capabilities to image extended radio sources.

\section{Simulation overview}

The codes for the simulation of imaging model extended source and its observation with the GMRT were written using Python and the CASA tool-kit [10, 4]. The following steps were carried out:

1. A model sky containing a source of Gaussian shape was made. This was assumed to be the only source in the sky. The code was written to have the flexibility to allow choice of position in the sky, the width and strength of the Gaussian source.

2. The GMRT array configuration with the positions of the antennas in the local coordinate system with the antenna C02 as reference was used. The position of the reference antenna C02 on Earth was taken as listed in CASA.

3. The observing frequency, bandwidth, number of channels in the bandwidth, total observation duration and separation between scans were set according to the particular simulation experiment.

4. The simulated observation was then imaged using the task 'clean' in CASA (details in Sec. 2.3)

5. The cleaned image was compared with the model image.

The aim of the simulations is to quantify the recovery of extended source such as a radio halo when the uv-coverage is varied. The results of the morphology and flux density recovery varying the following parameters are presented:

1. source angular size,

2. source flux density,

3. observation duration,

4. source declination, and

5. bandwidth.

The case of "source flux density" variation is considered in order to demonstrate that the recovery does not depend on the source strength. Thus the results from the rest of the cases can be considered general enough to be applicable to source strengths other than that chosen here.

\subsection{Observing parameters}

We made single channel visibilities of width $1 \mathrm{MHz}$ as observed with the GMRT at $610 \mathrm{MHz}$ to study the recovery as a function of observing duration, source strength and position. For the bandwidth case, we simulated multichannel (each channel of $1 \mathrm{MHz}$ ) visibilities of bandwidth $33 \mathrm{MHz}, 100 \mathrm{MHz}$ and $200 \mathrm{MHz}$ in the frequency band of 250-500 MHz. Simulated observations with a bandwidth of $33 \mathrm{MHz}$ represents GMRT whereas observations with a bandwidth of $100 \mathrm{MHz}$ and $200 \mathrm{MHz}$ represents observations with the U-GMRT. Integration time was taken to be 16 seconds in case of GMRT observation and 8 seconds in the case of the UGMRT. The observation duration is two hours starting at the transit time of the source in the cases of angular size, source flux density and declination. In the case of varying observing duration, multi-scan observations and bandwidth the start time of the observations is fixed to the rise time of the source.

\subsection{Source model}

The motivation for our simulation study comes from the limitations of interferometers that are faced in imaging the extended sources towards galaxy clusters. Therefore we discuss the choice of our model based on the case of a radio halo though it is applicable to extended source of any origin. Radio halos are Mpc sized diffuse sources associated with the intra-cluster medium of merging galaxy clusters [e. g. 7] and are among the most challenging to image. We chose a 2D Gaussian as a simplistic representation of a radio halo. The linear size of our model halo was taken as $1 \mathrm{Mpc}$ which corresponds to the FWHM of the chosen Gaussian and variation in its angular size represented the observation of radio halo at different redshifts. For all the single channel studies, model image was made at $610 \mathrm{MHz}$. We referred to the radio halo in the galaxy cluster Abell 2163 [6] for the realistic estimation of the total flux density $(S)$ for our model source and found it to be 0.6 $J y$. Model was also made over a range of redshifts $(z)$ from 0.05 to 1.0 and its angular size $(\theta)$ was calculated as a function of $z$ using the $\Lambda$-CDM cosmology [5]. We chose $S$ for a model at $\mathrm{z}=0.05$ to be $0.6 \mathrm{Jy}$ and it was scaled according to the redshift. The $\theta$ and $S$ values of the model to the corresponding $z$ are given in Table 1 . The source size of $1 \mathrm{Mpc}$ at the redshift of 0.05 corresponds to the largest angular size sampled at 610 $\mathrm{MHz}$ with the GMRT $\left(\lambda / B_{\min }\right)$. Position of the model source was taken as $(\mathrm{RA}, \mathrm{Dec}) \equiv\left(4 \mathrm{~h}, 60^{\circ}\right)$ for all but the case where the declination was varied.

\subsection{Using 'clean' in CASA}

CASA version 4.5.1 was used for analysis. Simulated visibilities were imaged using the task 'clean'. Since 

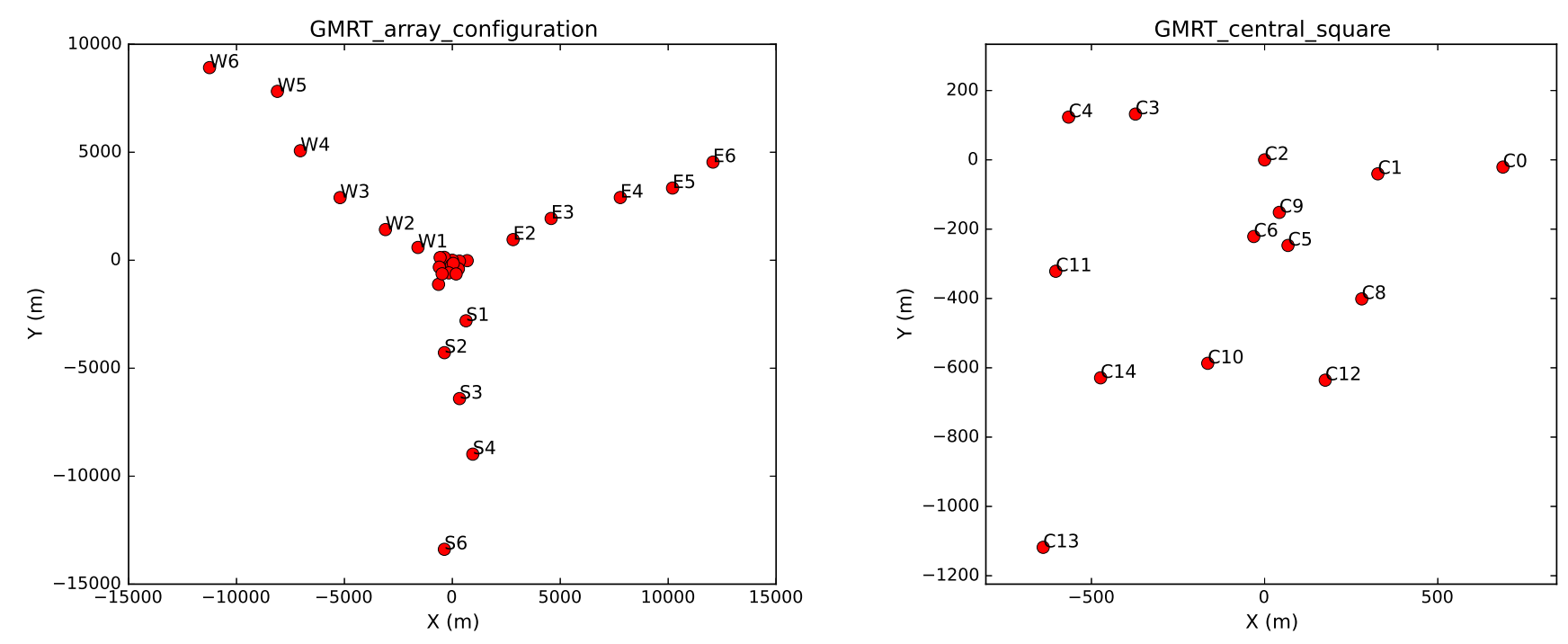

Fig. 1 Left - GMRT array configuration in local reference frame with the origin at the central square antenna C02. The arm antennas are labelled. Y axis is towards local north and X axis is towards local east. Right - The central square containing 14 antennas is shown with the antenna labels.

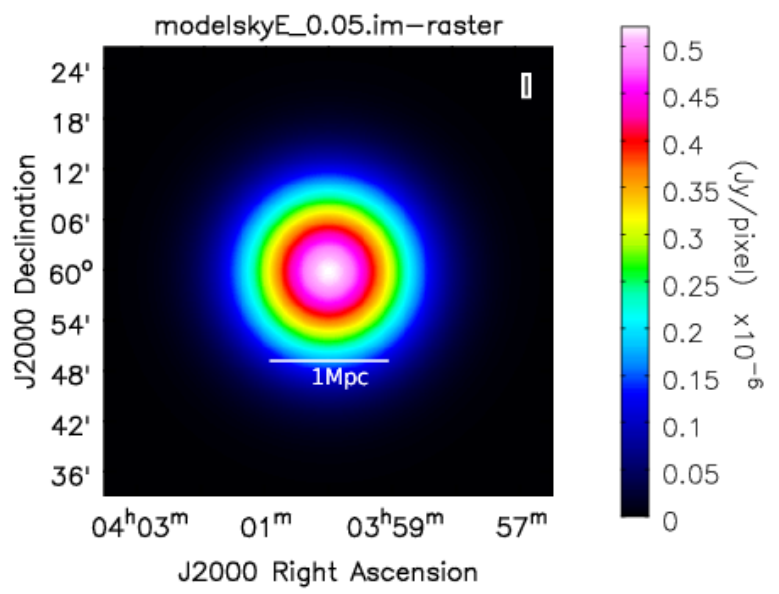

Fig. 2 2D Gaussian of width $16.8^{\prime}$ at FWHM representing a radio halo of size $1 \mathrm{Mpc}$ at the redshift of 0.05 . Source is centred at position $\left(4 \mathrm{~h}, 60^{\circ}\right)$. Total flux density $(S)$ of the source is $0.6 \mathrm{Jy}$. The color scale shows the flux density in the units of $J y$ pixel $^{-1}$.

imaging of extended sources is involved, we used the option of multi-scale cleaning. The multi-scale parameter in clean was set to have four values that were 0 , twice and ten times the synthesised beam and the fourth one equal to the angular size of the model source. Image size was taken as twice the size of the primary beam of GMRT. Cell size was chosen to be 0.25 times the synthesised beam. Cleaning was done interactively until no unique peak could be identified visually - this is when the residuals look uniform over the image. In this process an error is introduced based on where we stop the cleaning based on visual inspection (see Sec. 2.4).
Table 1 Angular size $(\theta)$ and total flux density $(S)$ of the source model at a particular redshift $(z)$.

\begin{tabular}{lll}
\hline$z$ & $\theta\left({ }^{\prime}\right)$ & $S(J y)$ \\
\hline 0.05 & 16.8 & 0.6000 \\
0.10 & 9.0 & 0.1441 \\
0.13 & 7.3 & 0.0832 \\
0.15 & 6.4 & 0.0616 \\
0.17 & 5.9 & 0.0472 \\
0.2 & 5.2 & 0.0333 \\
0.3 & 3.9 & 0.0138 \\
0.4 & 3.3 & 0.0072 \\
0.5 & 2.9 & 0.0043 \\
0.6 & 2.7 & 0.0028 \\
0.8 & 2.4 & 0.0014 \\
0.9 & 2.3 & 0.0010 \\
1.0 & 2.2 & 0.0008 \\
\hline
\end{tabular}

\subsection{Diagnostics for comparison}

The recovery of the model image in the cleaned image was compared on the basis of the total flux density recovery $\left(S_{\text {recov }}\right)$ and the morphology recovery $\left(M_{\text {recov }}\right)$.

Flux density recovery The total flux density of the cleaned image $\left(S_{\text {cleaned }}\right)$ of the source was compared to that of the model $\left(S_{\text {model }}\right)$ as:

$S_{\text {recov }}=\frac{S_{\text {cleaned }}}{S_{\text {model }}} \times 100$.

The recovered flux density after 'clean' has an error introduced due to the interactive cleaning. We examined the flux density and the value of peak in the residual images as measures of uncertainty on the recovered 


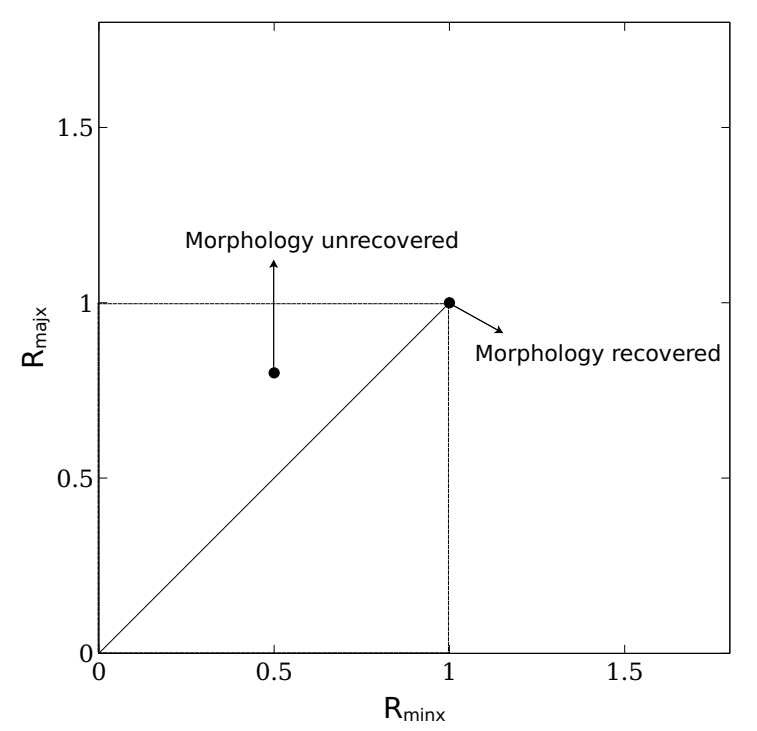

Fig. 3 Schematic representation of morphology recovery. A value at $(1,1)$ implies that the morphology of the source has been completely recovered and any deviation from it along the line $R_{\operatorname{majx}}=R_{\operatorname{minx}}$ or $R_{\operatorname{majx}}>R_{\operatorname{minx}}$ implies incomplete recovery. No values will be seen in the region $R_{\operatorname{majx}}<R_{\operatorname{minx}}$ as by definition, the major axis is greater than the minor axis.

cleaned flux density. The residual flux density is $0.1-8 \%$ and the peak residual flux density is $0.1-16 \%$ of that in the model image. This can be considered as the typical uncertainty on the reported $S_{\text {recov }}$.

Morphology recovery The model image is a circularly symmetric 2D Gaussian. The departure from the circularity serves as a quantitative estimate of the recovered morphology. Gaussian fit was done over the cleaned and model images of the source to determine the shape (major and minor axes). $M_{\text {recov }}$ was quantified by comparing the ratio of the major axis of the cleaned image to that of the model $\left(R_{m a j x}\right)$ with the ratio of their minor axes $\left(R_{\min x}\right)$ (Fig 3$)$. The location of the point in the $R_{\text {majx }}-R_{\min x}$ plane quantifies its departure from the model circular 2D Gaussian. An incomplete morphology recovery can also result in a value greater than $(1,1)$ in the plane when the recovered Gaussian is more extended with a slight decrease in its peak value. Error on the $M_{\text {recov }}$ is as reported on Gaussian fits in CASA. This is between $0.01-0.1 \%$ in all the cases and is not plotted in the figures.

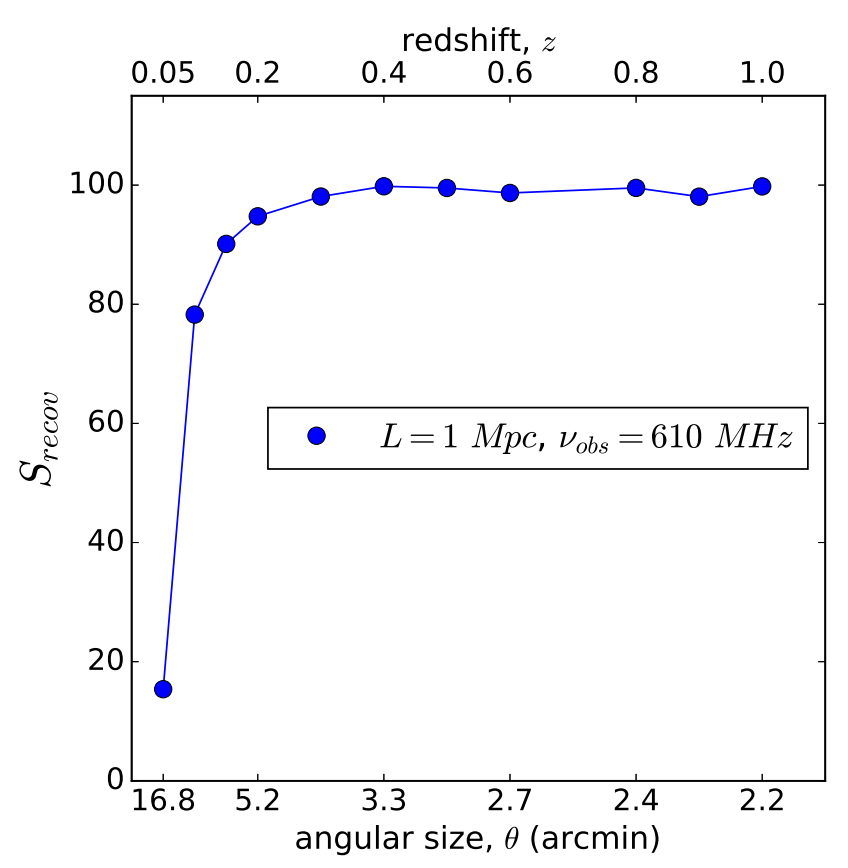

Fig. $4 S_{\text {recov }}$ as a function of $\theta(z)$ at $610 \mathrm{MHz}$ GMRT observation. Source with $\theta=16.8^{\prime}$, largest $\theta$ that can be sampled at $610 \mathrm{MHz}$ from GMRT, shows $S_{\text {recov }} \sim 14 \%$. $S_{\text {recov }} \sim$ $100 \%$ for the sources with $\theta<5.2^{\prime}$ or which are at $z>0.2$.

\section{Results}

\subsection{Angular size}

The model source image of different angular sizes was made by varying $z$ from 0.05 to 1.0 . In each case, an observation at $610 \mathrm{MHz}$ for 2 hours starting at the source transit time was carried out. $S_{\text {recov }} \sim 100 \%$ was observed for the sources with $\theta<5.2^{\prime}$ or which were at $z>0.2$ (Fig. 4). Therefore in the remaining cases, we restrict to the angular size range $>5.2^{\prime}$.

\subsection{Source flux density}

The strength of the model source scaled by factors of two, three and 100 times that of that at $z=0.05$ and kept rest of the observation parameters same as in the angular size case (section 3.1). The $S_{\text {recov }}$ and $M_{\text {recov }}$ of the source remains unchanged with increase in source strength (Fig. 5) basically due to the unchanged uv-coverage. Sources with $\theta=16.8^{\prime}$ shows $S_{\text {recov }}$ of $14 \%$ whereas $9^{\prime}$ sources shows $\sim 90 \%$ recovery. For $9^{\prime}$ sources, $M_{\text {recov }}$ is $\sim 90 \%$ whereas $16.8^{\prime}$ sources shows $73 \%$ recovery in their major axis while $63 \%$ recovery in their minor axis. 

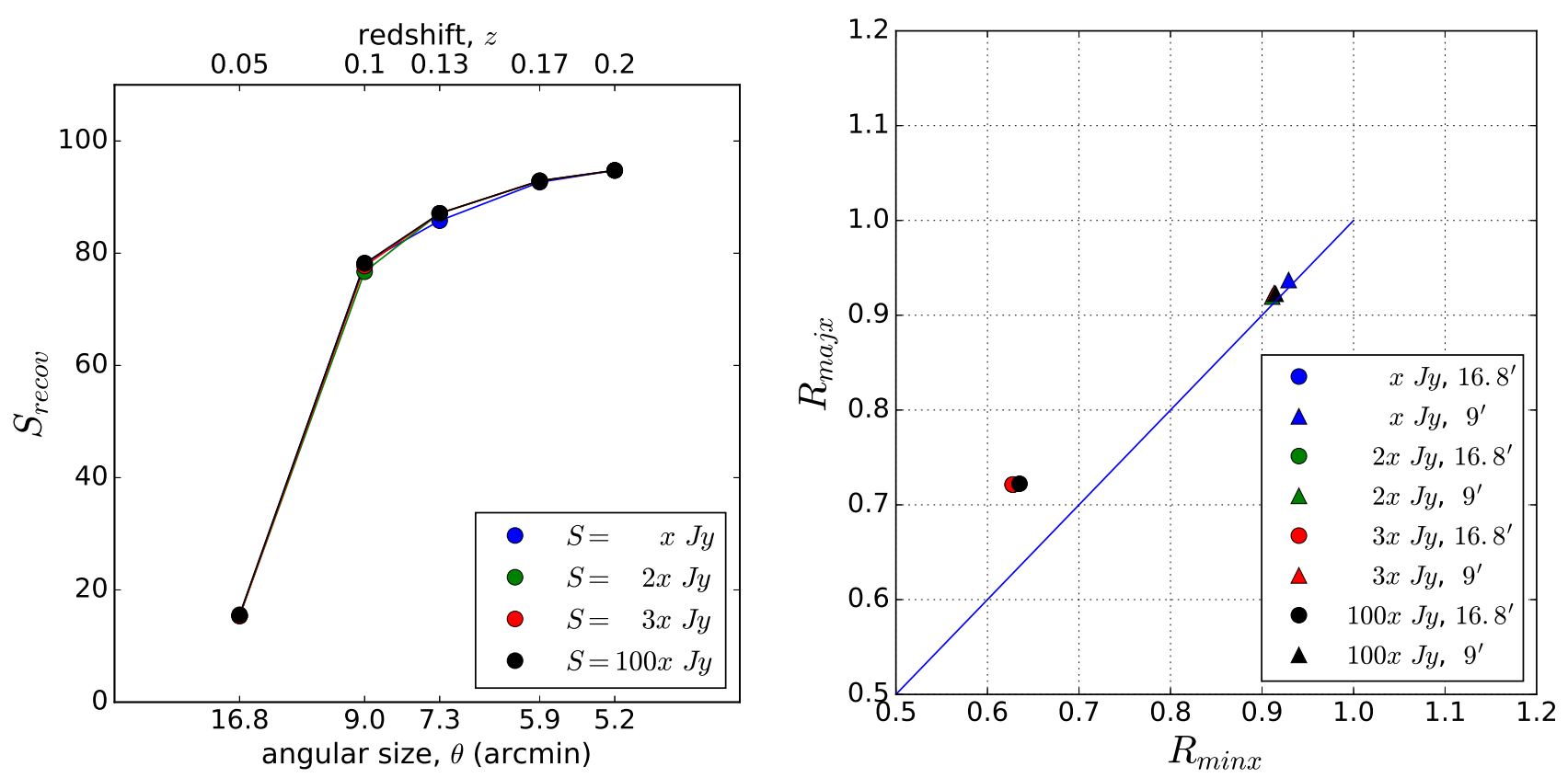

Fig. 5 Flux density case: Left $-S_{\text {recov }}$ when total flux density $(S)$ of the source is varied from $x=0.6$ Jy to $2 x, 3 x$ and $100 x$ Jy. $S_{\text {recov }}$ doesn't vary with varying $S$. Right $-M_{\text {recov }}$ for the sources with $\theta=16.8^{\prime}$ and $9^{\prime}$ when the source flux density is varied. $9^{\prime}$ sources shows $\sim 90 \%$ recovery in morphology whereas $16.8^{\prime}$ sources $73 \%$ recovery in their major axis while $63 \%$ recovery in their minor axis.

\subsection{Observing duration}

The simulation was carried out with observing durations of 2, 4, 6 and 12 hours, keeping the strength, position of the source, observing frequency and bandwidth constant. In all these cases the start time of the observation was chosen as the rise time of the source. The maximum time 12 hours, is the duration of full synthesis observation for the source at the chosen position. $S_{\text {recov }}$ increases with the observation duration (Fig. 6, Left ). For a source with $\theta=16.8^{\prime}, S_{\text {recov }}$ increased from $\sim 60 \%$ in 2 hours (from rise time) to $74 \%$ in 12 hours observation. Significant improvement is seen in the case of $M_{\text {recov }}$ (Fig. 6, Right ). For $16.8^{\prime}$ source, $M_{\text {recov }}$ increased from $\sim 70 \%$ in 2 hours to $90 \%$ in 12 hours. Source with $\theta=9^{\prime}$ showed $92 \%$ recovery in 2 hours, $97 \%$ in 4 hours and complete $100 \%$ morphology recovery in 6 hours and 12 hours of observation.

\subsubsection{Multi-scan observation}

A short total observing time spread in short scans over a longer duration is possible. This is a common strategy to maximise the uv-coverage in a given total time on the source. The 2 hours observation was spread in 6 discrete scans of 20 minutes each at regular intervals spanning over 12 hours. Comparison was done only for the two largest angular size sources $\left(16.8^{\prime}\right.$ and $\left.9^{\prime}\right)$. $S_{\text {recov }}$ and $M_{\text {recov }}$ are similar to the continuous 12 hours observa- tion (Fig. 7). $S_{\text {recov }}$ for $\theta=16.8^{\prime}$ and $\theta=9^{\prime}$ is $\sim 74 \%$ and $92 \%$ respectively which is equivalent to the corresponding recoveries at 12 hours observation. $M_{\text {recov }}$ is above $90 \%$ for multi-scan 2 hours case and 12 hours case for $16.8^{\prime}$ source, $9^{\prime}$ source shows complete recovery for both the cases.

\subsection{Declination}

The distribution of baseline lengths changes as a function of declination due to projection. We chose declinations $60^{\circ}, 0^{\circ},-30^{\circ}$ and $-50^{\circ}$ that span the observing declination range of GMRT and studied the recoveries for a 2 hours observation from transit. At lower declinations (below $-30^{\circ}$ ), we have better $S_{\text {recov }}$ (Fig. 8, Left ) and $M_{\text {recov }}$ (Fig. 8, Right ). For source with $\theta=16.8^{\prime}$, $S_{\text {recov }}$ at $0^{\circ}$ and $60^{\circ}$ is $<30 \%$ whereas it is $\sim 80 \%$ below $-30^{\circ}$. For all the declinations, source with $\theta=9^{\prime}$ shows $>90 \% M_{\text {recov }}$. In case of $16.8^{\prime}$ source, $M_{\text {recov }}$ is $>90 \%$ at $-30^{\circ}$ and $-50^{\circ}$. At $60^{\circ}$, it recovers its shape by $\sim 70 \%$ and $\sim 60 \%$ along its major axis and minor axis respectively. At $0^{\circ}$, it shows $M_{\text {recov }}$ of $\sim 90 \%$ and $\sim 65 \%$ along its major axis and minor axis respectively.

\subsection{Bandwidth: GMRT and U-GMRT}

We made multichannel observations having instantaneous bandwidths of $33 \mathrm{MHz}$ representing the GMRT, 

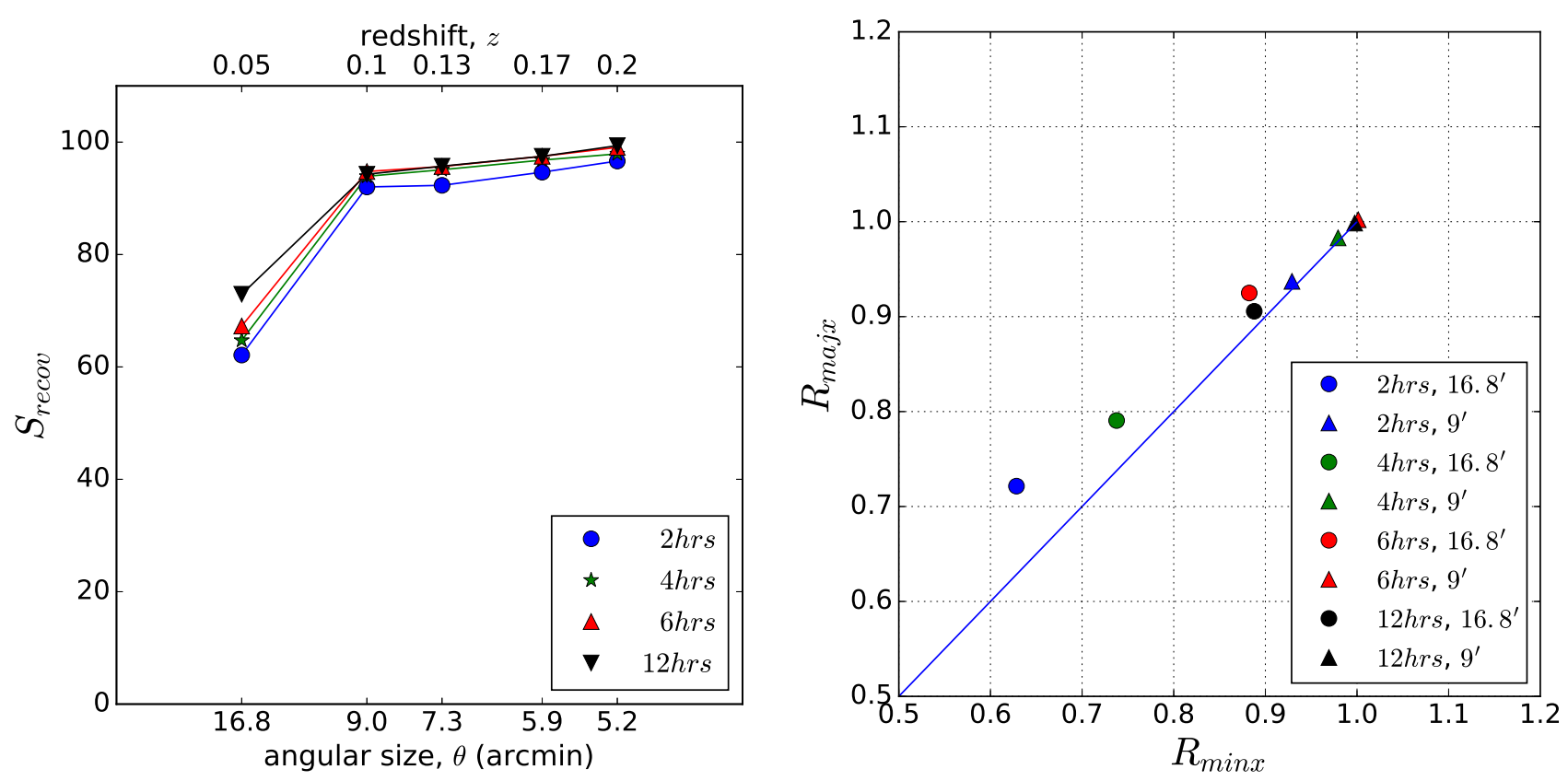

Fig. 6 Observation duration case: Left $-S_{\text {recov }}$ when observation duration is varied. Source with $\theta=16.8^{\prime}$ shows increase in its $S_{\text {recov }}$ from $\sim 60 \%$ in 2 hours to $73 \%$ in 12 hours observation. Right - Comparison of $M_{\text {recov }}$ when observation duration is varied. Source with $\theta=16.8^{\prime}$ shows increase in its $M_{\text {recov }}$ from $\sim 70 \%$ in 2 hours to $90 \%$ in 12 hours observation. Source with $\theta=9^{\prime}$ shows $92 \% M_{\text {recov }}$ in 2 hours and attains complete $100 \% M_{\text {recov }}$ in 6 hours of observation.
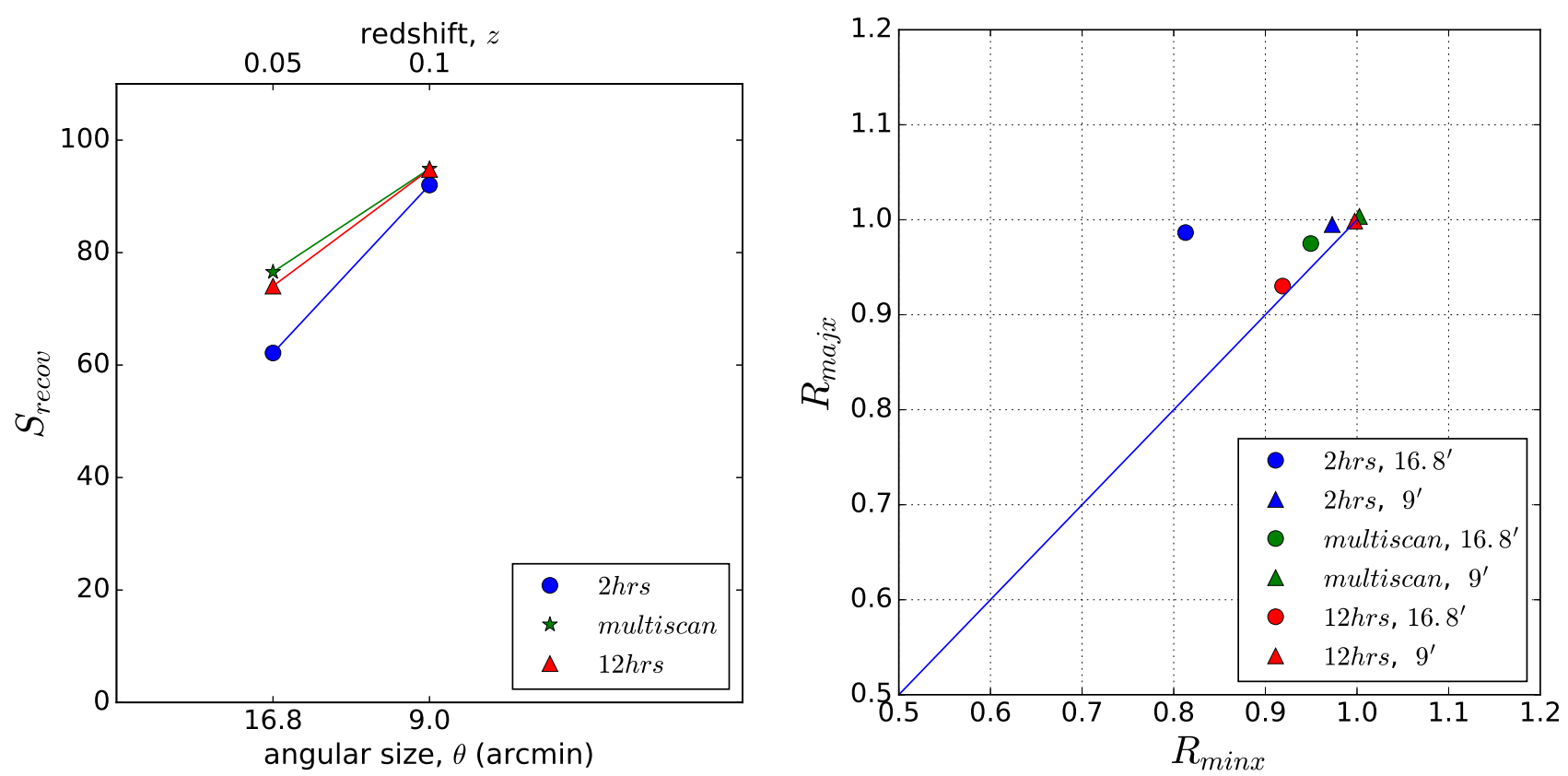

Fig. 7 Multi-scan case: Left $-S_{\text {recov }}$ comparison of the multi-scan 2 hours observation to continuous 2 hours and 12 hours observation case. $S_{\text {recov }}$ is similar for both multi-scan and 12 hours observation case. It is $\sim 77 \%$ for $16.8^{\prime}$ source and $92 \%$ for $9^{\prime}$ source. Right $-M_{\text {recov }}$ comparison of multi-scan 2 hours observation to continuous 2 hours and 12 hours observation case. $9^{\prime}$ source shows $\sim 100 \%$ recovery for all the 3 cases. $16.8^{\prime}$ source shows above $90 \%$ recovery in case of multi-scan and 12 hours observation whereas in case of 2 hours observation, $16.8^{\prime}$ source shows $\sim 100 \%$ recovery in its major axis but only $80 \%$ in its minor axis thus attaining an elongated shape along its major axis. 

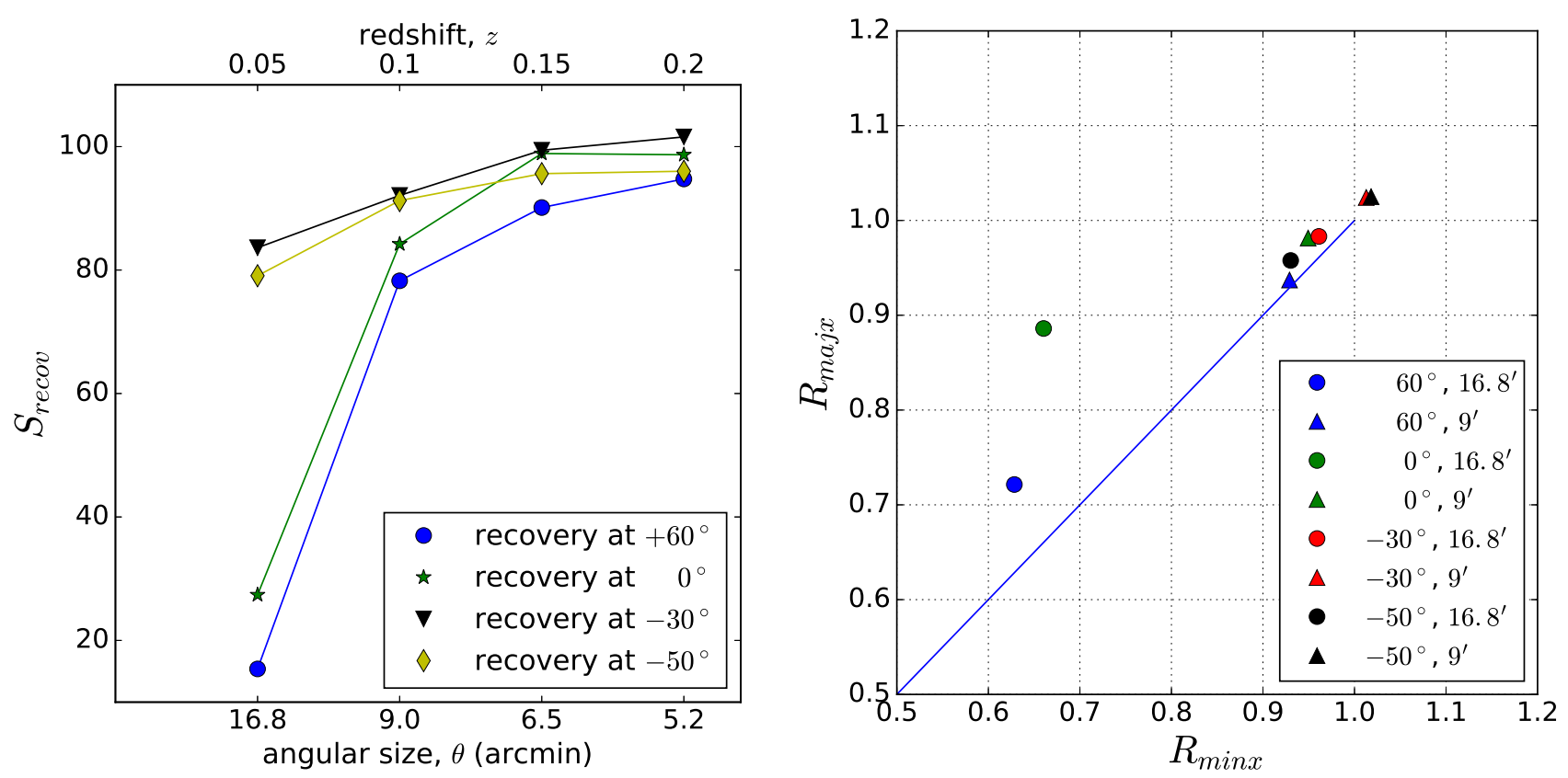

Fig. 8 Declination case: Left $-S_{\text {recov }}$ when source declination is varied. $S_{\text {recov }}$ is $<30 \%$ for source with $\theta=16.8^{\prime}$ at $0^{\circ}$ and $60^{\circ}$ and it is $\sim 80 \%$ for the same source at $-30^{\circ}$ and $-50^{\circ}$. Right $-M_{\text {recov }}$ when source declination is varied. For all declinations, $9^{\prime}$ source shows $M_{\text {recov }}>90 \%$. $16.8^{\prime}$ source shows $M_{\text {recov }}>90 \%$ at $-30^{\circ}$ and $-50^{\circ}$. At $60^{\circ} \& 0^{\circ}$, it recovers its shape by $\sim 70 \% \& \sim 90 \%$ along its major axis respectively and $\sim 60 \% \& \sim 65 \%$ along its minor axis respectively.

$100 \mathrm{MHz}$ and $200 \mathrm{MHz}$ representing the U-GMRT. The starting frequency in all these cases was fixed to 300 $\mathrm{MHz}$. Observations were carried out for 2 hours from rise of the source. Increase in bandwidth (BW) increases the $S_{\text {recov }}$ significantly and U-GMRT $(\mathrm{BW}=200 \mathrm{MHz})$ shows $\sim 90 \%$ flux density recovery as compared to that of $45 \%$ from GMRT even for the source with the largest angular size $\left(\theta=33.6^{\prime}\right)$ that can be sampled at 300 $\mathrm{MHz}$ (Fig. 9, Left ). Increasing bandwidth also improves the recovery in morphology. At $200 \mathrm{MHz}$, source with $\theta=33.6^{\prime}$ shows $98 \%$ recovery in major axis as compared to that of $90 \%$ at $33 \mathrm{MHz}$, recovery in minor axis increases from $76 \%$ to $92 \%$. For $16.8^{\prime}$ source, GMRT and U-GMRT both shows $M_{\text {recov }}>90 \%$ (Fig. 9, Right ) .

\section{Discussion}

Imaging of the sky with radio interferometers is a challenge due to the fact that the measurements are limited to the sampled angular scales and the sky distribution is an unknown. Therefore simulations of observations and imaging of extended sources are important to understand the limitations of the interferometer.

In this work, we presented the simulations of observations of a 2D Gaussian source with the GMRT at 610 $\mathrm{MHz}$. Observations were simulated with varying source declination, angular size, observing duration and band- width. The $33 \mathrm{MHz}$ GMRT observation was compared to the U-GMRT $200 \mathrm{MHz}$ bandwidth observation. The changes in the parameters reflected the change in the uv-coverage. The recovery of the total flux density and morphology of the source was quantified in each of the cases. The observed visibilities were chosen to be free of random noise in order to isolate the effect of the uv-coverage on the recovery of the model source after "cleaning".

\subsection{A rule of thumb for extended source recovery}

The simulations were carried out at the fiducial frequency of $610 \mathrm{MHz}$. The results obtained in the case of changing source declination, observation duration and source angular size can be scaled for interpretation at any other frequency. A recovery of $>90 \%$ was obtained for sources of angular size $5.2^{\prime}$ at $610 \mathrm{MHz}$ in 2 hours. The ratio $5.2^{\prime} / 16.8^{\prime}=0.3$ can be used to find the angular size at other frequency. For example, at $300 \mathrm{MHz}$ the largest angular scale sampled with the GMRT is $33.6^{\prime}$. The angular size at which the source will be fully recovered is $0.3 \times 33.6^{\prime}=10.1^{\prime}$, which can be also seen to be true from the simulation for the $33 \mathrm{MHz}$ bandwidth case shown in Fig. 9 (left). Therefore if $\theta_{\text {lar }}$ is the largest angular scale sampled with the GMRT at a given frequency, then near complete recovery sources of angular size, $\theta_{\text {recov }}=0.3 \times \theta_{\text {lar }}$ is possible. This can be 

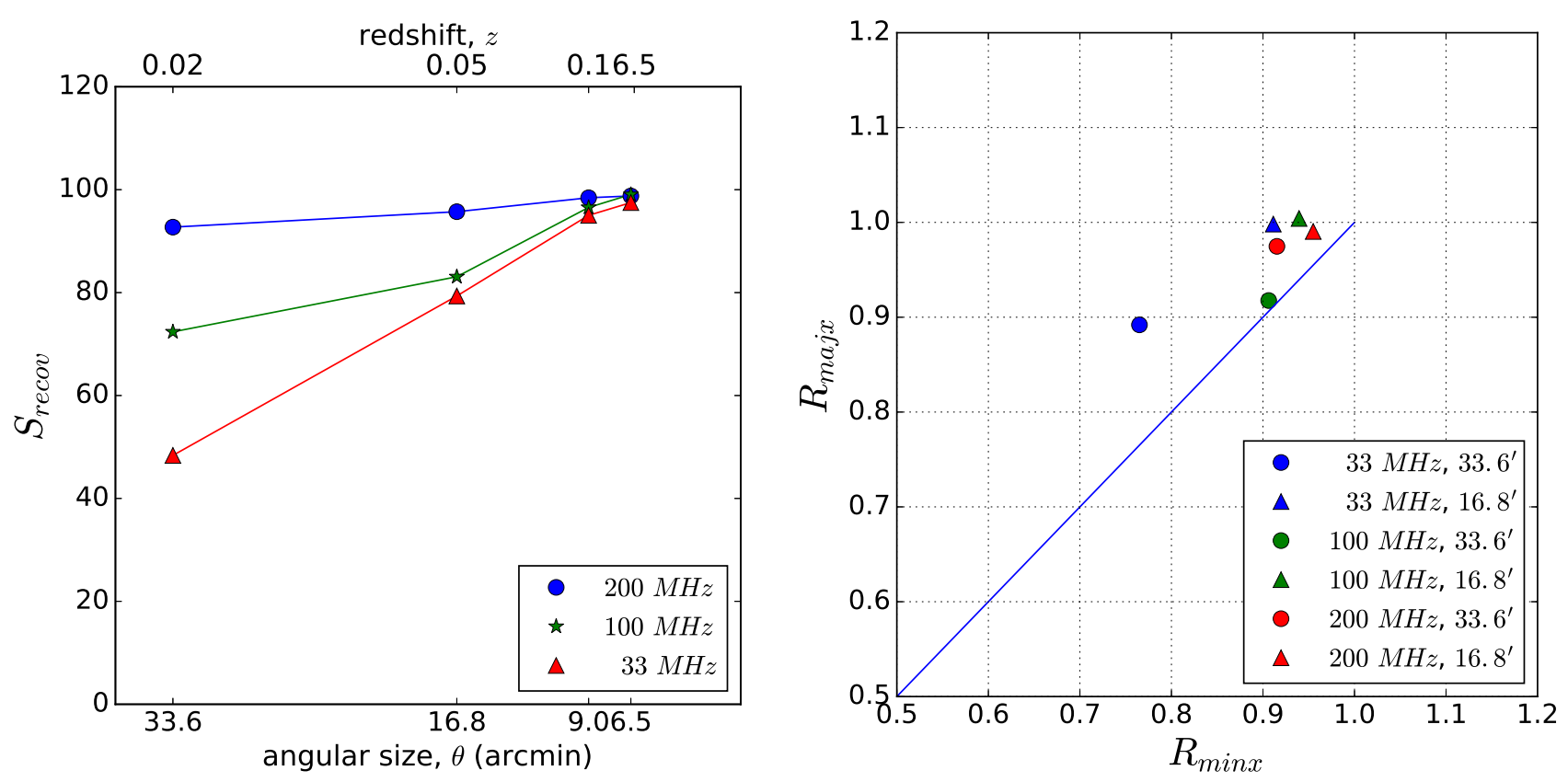

Fig. 9 Bandwidth case: Left $-S_{\text {recov }}$ when observation bandwidth is varied. $S_{\text {recov }}$ in case of $200 \mathrm{MHz}$ bandwidth (UGMRT) increases by a factor of 2 as compared to that of at $33 \mathrm{MHz}$ bandwidth (GMRT) while observing $33.6^{\prime}$ source which is the largest $\theta$ that can be sampled at $300 \mathrm{MHz}$ from GMRT. Right $-M_{\text {recov }}$ when observation bandwidth is varied. Sources observed at U-GMRT bandwidth (100 \& $200 \mathrm{MHz})$ shows $M_{\text {recov }}>90 \%$.

considered as a thumb rule for estimating the approximate scale at which a recovery above $80 \%$ is possible in a short observation ( $\sim 2$ hours).

The simulations presented here are applicable in general to the recovery of extended sources with an interferometer with uv-coverage similar to that of the GMRT. We illustrate the use of our results with a particular case of surveys that target search of extended sources of $1 \mathrm{Mpc}$ size in galaxy clusters. The redshift where the angular sizes correspond to the size of $1 \mathrm{Mpc}$ are plotted in Figures 4 - 9. If a target is located at declination $\delta_{s r c}$ at a redshift of $z_{s r c}$ and is planned to be observed for a duration of $t_{s r c}$ with a bandwidth $\Delta \nu$, then the recovery of a $1 \mathrm{Mpc}$ source of Gaussian type profile can be located in the plots. This can serve as a first step to check the feasibility of the survey in a given redshift range for objects of certain sizes with Gaussian profiles.

\subsection{Recommendations for observing strategies}

The results of this simulation can be used in deciding the strategies for targeted surveys for the search of diffuse radio emission of large angular scales. The recommendations for observing strategies are as follows:

- Cycling through sources to improve uv-coverage: From the simulation of a 2 hours observation divided into multiple scans spread over twelve hours it was shown that the improvement in uv-coverage provides a recovery of extended source nearly as good as a twelve hour observation (Sec. 3.3.1, Fig. 7). This strategy is often used when there are multiple targets in the sky that can be cycled through. An example of this strategy is the Australia Telescope Low-Brightness Survey [11].

- Low elevation observations: The shortest projected baselines of an array are when the targets are observed at the lowest possible elevation. The density of short baselines goes up as one observes a target at low elevation. For the GMRT the number of visibilities within $0-5 k \lambda$ can be a factor of 1.5 higher for targets with declinations $-50^{\circ}$ as compared to those at declinations $60^{\circ}$ (Fig. 10). The low declination sources represent targets observed at low elevations for the GMRT. If a target is observed for a limited duration, then in order to have the best chance of recovering all the extended emission, the observation be done either at the rise or setting time of the source when the lowest elevation data can be recorded. Observing at low declinations can be disfavoured for other reasons. At the GMRT one of the concerns with low elevation observing is the higher level of radio frequency interference coming in through terrestrial sources. 
- Long observations: Full synthesis observations are needed in order to recover the morphology of the source. A full synthesis observation is recommended if the angular size of the source to be recovered is close to that of the largest angular scale sampled by the interferometer.

- Wide bandwidths: U-GMRT is going to be a wide band system offering instantaneous bandwidths of $200-400 \mathrm{MHz}$ over fixed frequency bands in the range 120 - $1500 \mathrm{MHz}$. Wider bandwidths imply radially broad tracks in the uv-plane resulting in better uv-coverage. For a model source with $\theta=33.6^{\prime}$ we recovered $\sim 90 \%$ of the source flux density from $200 \mathrm{MHz}$ bandwidth observation as compared to $70 \%$ from the $100 \mathrm{MHz}$ bandwidth and $45 \%$ from the $33 \mathrm{MHz}$ bandwidth observation.

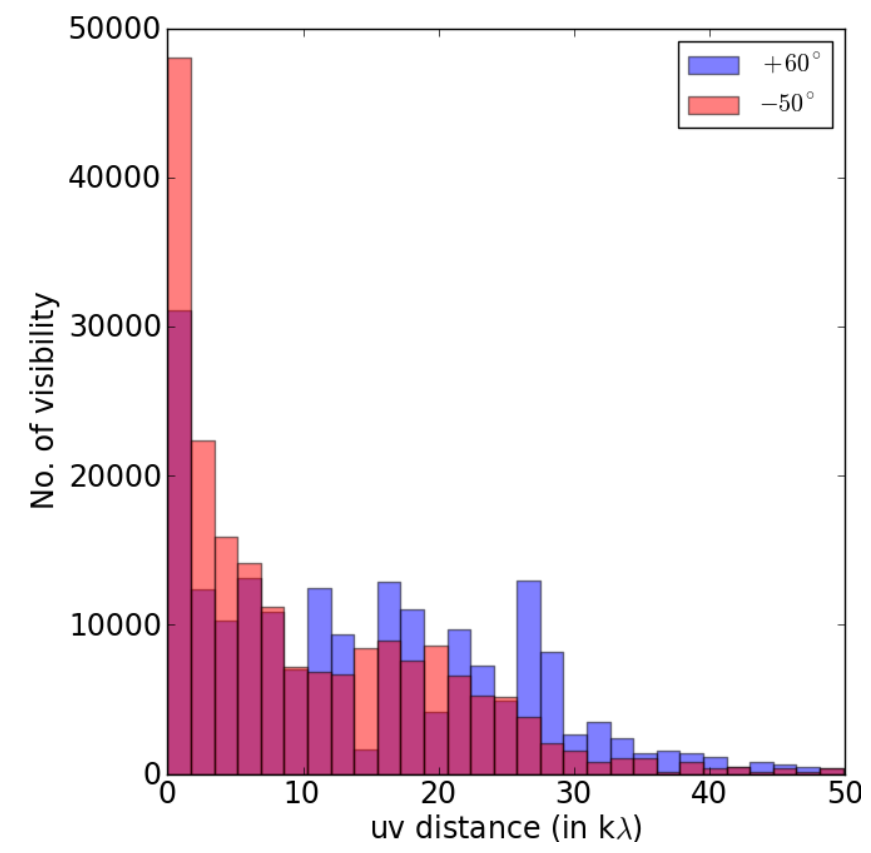

Fig. 10 Visibility distribution comparison of the source at $610 \mathrm{MHz}$ observation from GMRT at the declinations of $60^{\circ}$ and $-50^{\circ}$. Dark pink region is common to both the histograms.

\section{Conclusions}

We have simulated the observations of extended sources from GMRT and U-GMRT and quantified their limitations in the recovery in flux density and morphology of the sources when various parameters affecting the uv-coverage were varied. Results from this study are summarised below:
- $S_{\text {recov }}$ is $\sim 100 \%$ from GMRT for sources with $\theta<$ $5.2^{\prime}$ when observed for 2 hours from transit at 610 $\mathrm{MHz}$.

- $S_{\text {recov }}$ improves with increasing observation duration, however, improvement is insignificant due to the noise-free simulation of the observations. Improvement is more significant in the case of morphology recovery as compared to the flux density recovery. A 2 hours observation when spread over 12 hours (full synthesis) observation gives the similar recovery $\left(S_{\text {recov }} \& M_{\text {recov }}\right)$ as full synthesis observation.

- Low declination observation $<-30^{\circ}$ show near $80 \%$ recovery as compared to the higher declinations due to shorter projected baselines.

- $S_{\text {recov }}$ of the source with largest angular size that can be sampled in P-band $(300-500 \mathrm{MHz})$ is better by a factor of two from U-GMRT as compared to the GMRT.

Based on the simulations, as a thumb rule, in a GMRT observation at a given frequency, $>80 \%$ recovery of a source of angular size, $\theta_{\text {recov }}=0.3 \times \theta_{\text {lar }}$ is possible.

The simulations have quantified the recovery of extended sources with the GMRT and U-GMRT and can be used to plan observations. Such simulations can be carried out using the same codes for any interferometer such as the JVLA and the Square Kilometer Array (SKA).

Acknowledgements We thank the referee for the valuable comments. RK acknowledges the support through the DSTINSPIRE Faculty award. DKD is supported through the DSTINSPIRE project fund. We thank N. Mohan for insightful discussions. We thank D. Oberoi for providing the GMRT antenna coordinates. GMRT is run by the National Centre for Radio Astrophysics of the Tata Institute of Fundamental Research.

\section{References}

1. http://www.gmrt.ncra.tifr.res.in/

2. http://www.ncra.tifr.res.in:8081/ secr-ops/sch/c32web-

3. http://www.gmrt.ncra.tifr.res.in/gmrt_hpage/Upgrade/i

4. https://casa.nrao.edu/docs/CasaRef/CasaRef .html

5. Ade, P.A.R., et al.: Planck 2015 results. XIII. Cosmological parameters. Astron. Astrophys. 594, A13 (2016). DOI 10.1051/0004-6361/201525830

6. Feretti, L., Fusco-Femiano, R., Giovannini, G., Govoni, F.: The giant radio halo in abell 2163. Astron. Astrophys. 373, 106 (2001). DOI 10.1051/ 0004-6361:20010581

7. Feretti, L., Giovannini, G., Govoni, F., Murgia, M.: Clusters of galaxies: observational properties of the 
diffuse radio emission. A\&A Rev.20, 54 (2012). DOI 10.1007/s00159-012-0054-z

8. Kale, R., Venturi, T., Giacintucci, S., Dallacasa, D., Cassano, R., Brunetti, G., Cuciti, V., Macario, G., Athreya, R.: The Extended GMRT Radio Halo Survey II: Further results and analysis of the full sample. Astron. Astrophys. 579, A92 (2015). DOI 10.1051/0004-6361/201525695

9. Kale, R., Venturi, T., Giacintucci, S., Dallacasa, D., Cassano, R., Brunetti, G., Macario, G., Athreya, R.: The Extended GMRT Radio Halo Survey I: New upper limits on radio halos and mini-halos. Astron. Astrophys. 557, A99 (2013). DOI 10.1051/ 0004-6361/201321515

10. McMullin, J.P., Waters, B., Schiebel, D., Young, W., Golap, K.: CASA Architecture and Applications. In: R.A. Shaw, F. Hill, D.J. Bell (eds.) Astronomical Data Analysis Software and Systems XVI, Astronomical Society of the Pacific Conference Series, vol. 376, p. 127 (2007)

11. Subrahmanyan, R., Ekers, R.D., Saripalli, L., Sadler, E.M.: ATLBS: the Australia Telescope Low-Brightness Survey. MNRAS 402, 2792-2806 (2010). DOI 10.1111/j.1365-2966.2009.16105.x

12. Swarup, G., Ananthakrishnan, S., Kapahi, V.K., Rao, A.P., Subrahmanya, C.R., Kulkarni, V.K.: The Giant Metre-wave Radio Telescope. Current Science 60(2), 95-105 (1991)

13. Thompson, A.R., Moran, J.M., Swenson, G.W.: Interferometry and Synthesis in Radio Astronomy; 2nd ed. Wiley-VCH, Weinheim (2001)

14. Venturi, T., Giacintucci, S., Brunetti, G., Cassano, R., Bardelli, S., Dallacasa, D., Setti, G.: GMRT Radio Halo Survey in galaxy clusters at $\mathrm{z}=0.2-0.4$. 1. The REFLEX sub-sample. Astron. Astrophys. (2006). DOI 10.1051/0004-6361:20065961. [Astron. Astrophys.463,937(2007)]

15. Venturi, T., Giacintucci, S., Dallacasa, D., Cassano, R., Brunetti, G., Bardelli, S., Setti, G.: GMRT Radio Halo Survey in galaxy clusters at $\mathrm{z}=0.2-0.4$. II.The eBCS clusters and analysis of the complete sample. Astron. Astrophys. 484, 327 (2008). DOI 10.1051/0004-6361:200809622

16. Venturi, T., Rossetti, M., Bardelli, S., Giacintucci, S., Dallacasa, D., Cornacchia, M., Kantharia, N.: Radio emission at the centre of the galaxy cluster Abell 3560: evidence for core sloshing? Astron. Astrophys. 558, A146 (2013). DOI 10.1051/ 0004-6361/201322023

17. Wilner, D.J., Welch, W.J.: The S140 core: Aperture synthesis $\mathrm{HCO}(+)$ and $\mathrm{SO}$ observations. ApJ 427, 898-913 (1994). DOI 10.1086/174195 